\title{
DESARROLLO DE MODELOS DE BIOMASA AÉREA EN SOMBRÍOS DE CAFETO (Coffea arabica L.) MEDIANTE DATOS SIMULADOS
}

\section{DEVELOPING ABOVE-GROUND BIOMASS MODELS FOR COFFEE (Coffea arabica L.) SHADE TREES VIA SIMULATED DATA}

\author{
Javier Darío Aristizábal ${ }^{1}$
}

\begin{abstract}
${ }^{1}$ Ingeniero Forestal. Especialista en Planeación Ambiental y Manejo Integrado de Recursos Naturales. Fundación Natura. Carrera 116B No. 77-67 Int. 7 Apto. 402. Bogotá D.C., Cundinamarca, Colombia.jaristizabal@natura.org.co
\end{abstract}

Rev. U.D.C.A Act. E Div. Cient. 14(1): 49 - 56, 2011

\section{RESUMEN}

Los modelos alométricos han sido una valiosa herramienta para predecir el crecimiento de la biomasa de diversas especies de árboles; sin embargo, dichos modelos han sido construidos para muy pocas especies. El desarrollo de ecuaciones alométricas para otras especies forestales trae consigo ciertas complejidades, desde el punto de vista logístico, operativo y de costos. La presente investigación propone el desarrollo de un modelo genérico, a partir de datos simulados, generados de ecuaciones alométricas construidas con datos reales de campo, el cual, puede ser aplicado para pronosticar el crecimiento de la biomasa de aquellas especies forestales que, tradicionalmente, son utilizadas como sombríos de cafeto u otro tipo de cultivo. De igual forma, se elaboró un modelo alométrico para el cafeto, siguiendo el mismo procedimiento. Para el caso de los árboles, la ecuación genérica fue construida con base en la información volumétrica de catorce especies de distintas edades. Los resultados de los modelos, tanto de árboles como de cafetos, muestran un comportamiento conservador y coherente en comparación con los datos reales de campo, lo que hace factible su aplicabilidad, para predecir la biomasa de los componentes.

Palabras clave: Modelo, alométrico, biomasa, árboles de sombra, agroforestal.

\section{SUMMARY}

Allometric models have been a valuable tool for predicting biomass growth of several tree species, however, such models have only been constructed for a few of them. Developing allometric models for other tree species implies certain complexities from the logistic, operational and cost based point of view. This research proposes the development of a generic allometric model through simulated data generated from allometric equations constructed from real field data that could be used for forecasting biomass growth of tree species usually planted as shade for coffee or other crops. Likewise, an allometric model for coffee bushes, following same procedure was made. In case of shade trees, the generic allometric equation was based on volumetric data of fourteen tree species. Results from models for both shade trees and coffee brushes show a conservative, coherent performance in comparison with real field data which allows its application in order to predict the components of biomass.

Key words: Model, allometric, biomass, shade trees, agroforestry.

\section{INTRODUCCIÓN}

Durante la última década, se han emprendido diferentes iniciativas en el sector AFOLU (Agriculture, Forestry and Other Land (Use), para contrarrestar los efectos del calentamiento global, provocado por el incremento de las concentraciones de gases de efecto invernadero en la atmósfera. En este sentido, las actividades agroforestales desempeñan un papel relevante, dado que se estima que el carbono almacenado por este uso de la tierra corresponde a aproximadamente el 12\% del carbono terrestre mundial (Smith et al. 1993 y Dixon 1995, citados por Albrecht E Kandji, 2003). 
En el trópico, el potencial de fijación de carbono por los sistemas agroforestales, se estima entre 12 a $228 \mathrm{Mg} \mathrm{ha}^{-1}$, dependiendo de la estructura del sistema y el medio en el que se establecen (Albrecht $\mathcal{E}$ Kandji, 2003). La distribución del carbono varía de acuerdo al depósito, pero la evidencia demuestra que el suelo contiene más del $60 \%$ respecto al total del carbono almacenado, seguido de la biomasa aérea, con el $30 \%$ y la necromasa con aproximadamente el $10 \%$ (Birdsey, 1992).

La inclusión de proyectos agroforestales, como mecanismo de mitigación de emisiones de gases de efecto invernadero (GEI), plantea ciertos retos metodológicos, que han sido abordados convenientemente por los diferentes cuerpos científicos internacionales, quienes han dado oportuna solución a los obstáculos presentados; no obstante, aún subsisten dificultades técnicas que impiden la implementación generalizada de proyectos de compensación de emisiones por parte de las comunidades rurales, quienes se podrían beneficiar aún más por la venta del carbono almacenado en sus fincas.

Entre dichas limitantes técnicas, se encuentra la insuficiencia de información relacionada con la producción de biomasa de las especies forestales, que son utilizadas localmente en los sistemas agroforestales, aspecto que cobra mayor relevancia en ciertas prácticas agrícolas, que por sus características deben contar con una importante cantidad de árboles, como parte del sistema. Este parece ser el caso de las fincas cafeteras o cacaoteras, que funcionan bajo esquemas de certificación, como la norma de la Red de Agricultura Sostenible, puesto que deben cumplir ciertos criterios en cuanto a la cantidad y el número mínimo de especies que deben ser plantados entre el cultivo principal (Rainforest Alliance, 2009).

Para predecir el incremento de la biomasa de un árbol, se utilizan métodos indirectos de estimación, como el uso de modelos alométricos o la aplicación de factores de expansión de biomasa (FEB) sobre existencias de volumen maderable.

El primer caso, se fundamenta en la alometría, que es definida como "las relaciones entre las dimensiones de los organismos y los cambios en las proporciones relativas de estas dimensiones con los cambios en el tamaño absoluto" (Goldman et al. 1990). En virtud de lo anterior y en el caso particular de un individuo arbóreo, un modelo alométrico es una ecuación matemática que relaciona la biomasa con variables del árbol, fácilmente medibles en pie, tales como el diámetro normal, altura de la copa libre, altura a la primera ramificación, altura total de la planta, ya sea en combinación simple o múltiple de estos atributos (Vallejo et al. 2008).

Teniendo en cuenta la alta heterogeneidad en cuanto a la composición del sombrío para cafetales o cacaotales es poco probable hallar ecuaciones alométricas específicas para todas las especies involucradas, salvo para aquellas especies que han sido extensamente documentadas, tal como Cordia alliodora (Ruíz y Pavón) Oken o Erythrina poeppigiana (Walp.) O. F. Cook (Ortiz et al. 2008; Segura et al. 2006; Oelbermann et al. 2004).

Ortiz et al. (2008) consideraron el uso de dos ecuaciones alométricas para $C$. alliodora, en dos sitios de Panamá, en donde la biomasa era predicha en función de la edad. Segura et al. (2006) desarrollaron modelos alométricos para C. alliodora, Inga punctata Willd., Inga tonduzzi Donn. Sm. y Juglans olanchana Standl. E L. O. Williams, en la provincia de Matagalpa, Nicaragua.

También, se han implementado modelos de alométricos de biomasa para Coffea arabica L., en Nicaragua (Segura et al. 2006; Suárez et al. 2002) e Indonesia (van Noordwijk et al. 2002).

El presente artículo plantea una solución alternativa a la elaboración de modelos alométricos de biomasa, basados en muestreos destructivos, al optar por construir una ecuación alométrica genérica, desarrollada a partir de datos simulados, originados a partir de modelos construidos con datos reales de campo. Para tal fin, fueron evaluados los sombríos utilizados en nueve fincas cafeteras, certificadas bajo el sello Rainforest Alliance y con base en la información dendrométrica obtenida, se diseño una ecuación genérica que pudiese ser aplicada a cualquier especie del sombrío.

\section{MATERIALES Y MÉTODOS}

Selección de fincas de acuerdo a la edad y establecimiento de parcelas: Para contar con árboles de diferentes clases diamétricas, se definieron los siguientes cinco rangos de edades: menor de cinco años, entre cinco y diez años, entre diez y 15, entre 15 y 20 y mayores de 20 años. Por cada rango, se escogieron dos fincas, seleccionadas de un núcleo de fincas cafeteras certificadas, del municipio de San Vicente de Chucurí (Departamento de Santander, Colombia).

En cada finca, se estableció una parcela temporal de 0,1ha $(10 \times 100 m)$ al interior del cafetal, en donde se midieron todos los árboles. De igual forma, cada parcela fue subdivida en 40 cuadrantes de $25 \mathrm{~m}^{2} \mathrm{y}$, aleatoriamente, fueron escogidos dos de ellos para medir los arbustos de cafeto.

Parámetros de medición: Para los árboles, se registraron las principales variables dendrométricas, que se consideran indispensables para efectuar cálculos de volumen, como el diámetro a la altura del pecho (DAP), la altura total, la altura fustal y el diámetro superior aparente. En este último caso, Pearson et al. (2005) sugieren el uso del relascopio, para determinar diámetros a diferentes alturas del fuste; sin embargo, una técnica mas simple consiste en el uso del 
método de la regla transparente, según, el cual, se observa el diámetro aparente del fuste a una altura determinada, a través de una regla transparente, la que se debe encontrar a una distancia fija de los ojos del observador (entre 10 a 20 centímetros). Se debe registrar la lectura del diámetro aparente y la distancia desde el punto de observación hasta el diámetro aparente. El diámetro real aproximado es el producto entre la lectura observada, a través de la regla y, el cociente, entre la distancia calculada entre el punto de observación hasta el árbol y la distancia entre el ojo y la regla. Todas las variables obligan utilizar las mismas unidades métricas.

En aquellos árboles cuya altura total no superaba los 1,3 metros, se registraba el diámetro apical. Para el caso de los arbustos de cafeto, se midió la altura total del tallo, el diámetro basal y el diámetro apical.

Las alturas de los árboles fueron estimadas utilizando un clinómetro (Suunto PM-5) y los cafetos fueron medidos con un flexómetro (Lufkin, ref.: L516CME). Para registrar los diámetros, se empleó cinta métrica, en el caso de los árboles y calibrador (vernier) (Mitutoyo, ref.: 530).

Cubicación de árboles y cafetos: Para la determinación de los volúmenes individuales, tanto de los árboles como de los arbustos de cafeto, se aplicó la ecuación volumétrica para conos truncados, sugerida por Pearson et al. (2007):

$V=\pi\left(R^{2}+r^{2}+R r\right) * h / 3 ;$ donde,

$\mathrm{V}=$ volumen del fuste $\left(\mathrm{m}^{3}\right)$

$\mathrm{R}=$ radio mayor $(\mathrm{m})$

$\mathrm{r}=$ radio menor $(\mathrm{m})$

$\mathrm{h}=$ altura del fuste hasta el diámetro superior aparente (m)

En el caso de los árboles, el radio mayor concernía al DAP $\mathrm{y}$, el menor, al diámetro superior aparente. Para los cafetos, el radio mayor correspondía al diámetro basal y el menor al diámetro apical.

Estimación de la biomasa aérea: Para calcular la biomasa aérea del sistema agroforestal de cafeto con sombrío diversificado fue necesario considerar un procedimiento que se resume en los siguientes pasos:

1. Conversión de volúmenes a biomasa seca: el volumen obtenido fue convertido a biomasa seca al multiplicarlo por la correspondiente densidad básica de la especie en cuestión. Dichas densidades aplican a las reportadas por Acero (1985). Para el caso del cafeto, se utilizó el dato de densidad indicado por Rominj E Wilderink (1987).

2. Determinación de factores de expansión de biomasa (FEB): se efectuó por inferencia lógica basada en ecuaciones alométricas elaboradas por otras investigaciones, a través de mediciones directas en campo. Tanto para los árboles de sombrío como para los cafetos, se emplearon cuatro ecuaciones alométricas, reportadas por Segura \& Andrade (2008), Segura et al. (2006), Suárez et al. (2002) y van Noordwijk et al. (2002). Con cada uno de ellos, se calculó la biomasa total, de acuerdo a la variable independiente utilizada (diámetro o altura), cuyos resultados fueron sumados y promediados para obtener un valor simulado de biomasa total (Cuadro 1). Este resultado fue dividido por el valor real de biomasa seca del fuste (tallo, en el caso de los cafetos), obtenido en la presente investigación y, finalmente, se obtuvo un FEB individual. El resultado fue correlacionado con la variable altura total (h), para los cafetos y DAP, para los árboles.

Para calcular el FEB de los árboles, se evaluó un total de 186 individuos, distribuidos entre las siguientes especies: Albizzia carbonaria Britton (cinco individuos),

Cuadro 1. Modelos alométricos utilizados para estimar los datos simulados.

\begin{tabular}{|c|c|c|c|}
\hline \multicolumn{3}{|c|}{ MODELO } & FUENTE \\
\hline \multirow{3}{*}{$\stackrel{\circ}{\stackrel{一}{\underline{L}}}$} & $\mathrm{~B}=10^{-1,0+(2 * \log (\mathrm{d} 15))+(0,54 * \log (\mathrm{h}))}$ & {$[1]$} & Segura et al. (2006) \\
\hline & $\mathrm{B}=10^{-1,2+(2,1 * \log (\mathrm{d} 15))}$ & {$[2]$} & Segura et al. (2006) \\
\hline & $\ln (B)=-2,39287+(0,95285 * \ln (d 15))+(1,2693 * \ln (h))$ & {$[4]$} & Suárez et al. (2002) \\
\hline \multirow{3}{*}{ 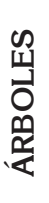 } & $\mathrm{B}=10^{-1,54+(2,05 * \log (\mathrm{dcm}))+(1,18 * \log (\mathrm{h}))}$ & [1] & Segura E Andrade (2008) \\
\hline & $\mathrm{B}=10^{-1,0+(2,3 * \log (\text { dap }))}$ & {$[3]$} & Segura et al. (2006) \\
\hline & $B=10^{-0,9578+(2,3408 * \log (\text { dap }))}$ & {$[4]$} & Suárez et al. (2002) \\
\hline
\end{tabular}

Notas: $\mathrm{B}=$ biomasa total $(\mathrm{kg}) ; \log =$ logaritmo base $10 ; \mathrm{h}=$ altura total $(\mathrm{m}) ; \mathrm{d} 15$ = diámetro del tronco a $15 \mathrm{~cm}$ de altura $(\mathrm{cm}) ;$ dap = diámetro a la altura del pecho $(\mathrm{cm}) ; \mathrm{ln}=$ logaritmo natural; dcm = diámetro cuadrático medio a una altura de $30 \mathrm{~cm}(\mathrm{~cm})$. 
Cecropia sp. (un individuo), Cedrela odorata L. (ocho individuos), Citrus sinensis Osbeck (cuatro individuos), C. alliodora (49 individuos), Erythrina edulis Triana ex Micheli (cuatro individuos), Erythrina fusca Lour. (diez individuos), E. poepiggiana (19 individuos), Inga codonantha Pittier (56 individuos), Myrsine ferruginea (Ruiz \& Pav.) Spreng (once individuos), Persea americana Mill. (un individuo), Tabebuia rosea (Bertol.) A.DC. (cuatro individuos), Trichantera gigantea (Bonpl.) (siete individuos) y Zanthoxylum rhoifolium Lam. (siete individuos).

Los resultados individuales de FEB fueron analizados estadísticamente, utilizando el programa STATGRAPHICS Plus 5, para determinar qué comportamiento presentaban con respecto al DAP y la edad, y de qué forma podían ser aplicados para obtener la biomasa total por individuo.

3. Elaboración de modelo alométricos: para el diseño de modelos de regresión se generaron seudodatos de biomasa seca total por individuo, aplicando los valores FEB sobre los datos de biomasa del fuste (o tallo), calculados previamente. Estos resultados fueron transformados para cumplir con los supuestos de normalidad y de homogeneidad de varianzas. Cada seudodato fue correla- cionado con su correspondiente variable predictora (diámetro o altura), para generar una ecuación de biomasa. Este paso, se aplicó tanto para los árboles de sombrío como para los arbustos de cafeto.

\section{RESULTADOS Y DISCUSIÓN}

Factor de expansión de biomasa para cafeto: El análisis de regresión arrojó como resultado, que el modelo de mejor ajuste era de tipo recíproco con respecto a $\mathrm{X}$ (variable independiente), el cual, presentó un coeficiente de determinación ajustado alto $\left(R^{2}{ }_{\text {ajust }}=0,96\right)$ y un error estándar de estimación bajo (RCME $=0,27)$. Los pronósticos del modelo son congruentes con lo expuesto por la literatura, en el sentido que, bajo condiciones de sombra, el FEB disminuye a medida que la variable independiente aumenta (IPCC, 2003; IPCC, 2006; Pearson et al. 2007; Vallejo et al. 2008). Con base en lo anterior, se pudo establecer un FEB variable para el cafeto con respecto a la altura de cada individuo. De acuerdo a la información derivada del modelo obtenido (Cuadro 2), se infiere que el FEB es drásticamente mayor cuando la altura del arbusto de cafeto era de 0,1 metros y cayó vertiginosamente hasta cuando alcanzó el

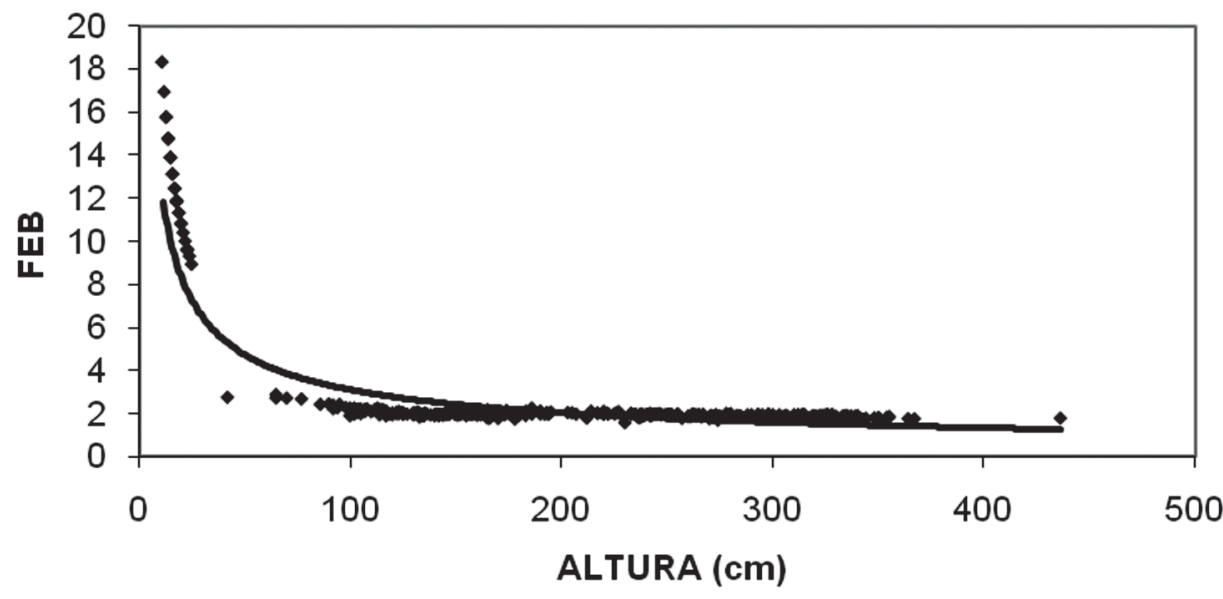

Figura 1. Relación alométrica entre el FEB y la altura total del cafeto (Coffea arabica L.).

Cuadro 2. Modelos alométrico para estimar FEB y biomasa en cafeto (Coffea arabica L.).

\begin{tabular}{|l|l|c|c|}
\hline \multicolumn{1}{|c|}{ PARÁMETRO } & \multicolumn{1}{c|}{ MODELO } & R $_{\text {AJust. }}$ & RCME \\
\hline FEB & FEB $_{\mathrm{i}}=0,981672+(189,052 / \mathrm{h})$ & 0,96 & 0,27 \\
\hline BIOMASA TOTAL & $\ln \left(\mathrm{B}_{\mathrm{t}}\right)=1,69001 * \ln (\mathrm{h})-8,78722$ & 0,85 & 0,41 \\
\hline FEB $_{\mathrm{i}}=$ factor de expansión de biomasa individual; $\quad \mathrm{h}=$ altura total $(\mathrm{m}) ; \quad \mathrm{ln}=$ logaritmo base e; $\quad \mathrm{B}_{\mathrm{t}}=$ Biomasa total $(\mathrm{kg})$
\end{tabular}


metro de altura. A partir de este punto, el FEB se estabilizó y se disminuyó gradualmente, cuando el cafeto presentó entre uno y tres metros de altura (Figura 1).

Modelo alométrico para cafeto a partir de datos simulados: Para estimar la biomasa total, se aplicó el valor de FEB resultante de la correspondiente ecuación sobre el valor de biomasa fustal individual. Con este valor, se construyó el modelo de crecimiento de biomasa total en función de la altura. Como resultado, se obtuvo que el modelo de mejor ajuste fue de tipo potencial con transformación lineal, logrando un coeficiente de determinación ajustado, que explica el $85 \%$ de la variable respuesta $\left(R^{2}{ }_{\text {jiust. }}=0,85\right)$ y un error estándar de estimación (RCME) de 0,41.

Con base en lo anterior, se puede inferir que la biomasa total de un arbusto de cafeto puede fluctuar desde 0,36kg para un individuo de un metro de altura hasta de $3,81 \mathrm{~kg}$, para un individuo de cuatro metros. Estos resultados son muy similares a los que se pueden obtener con las ecuaciones de Segura et al. (2006), cuyos valores fluctuarían entre 0,16 y $0,18 \mathrm{~kg}$, para un cafeto de un metro y 3,84 y 3,12kg, para un individuo de cuatro metros de altura. También son muy cercanos a los que se podrían obtener con la ecuación propuesta por Suárez et al. (2002) (0,17 y 3kg, para individuos de uno y cuatro metros, respectivamente); sin embargo, la principal diferencia se observa en el extremo inferior del rango, en donde la biomasa en cafetos de un metro, obtenida a partir de la ecuación propuesta, duplica el dato arrojado por las ecuaciones de los autores mencionados.

Factor de expansión de biomasa para árboles de sombrío: El FEB fue calculado individualmente como el cociente entre el dato promedio de biomasa total arrojado por las cuatro ecuaciones utilizadas, sobre el valor de biomasa fustal real, calculado como el producto entre el volumen fustal y la densidad básica de la especie correspondiente. Se trató de establecer si existía correlación entre el FEB y el DAP, pero el nivel de ajuste de los diferentes modelos probados fue muy bajo. En virtud de lo anterior, se analizó estadísticamente el comportamiento del FEB con respecto a la edad. El análisis de varianza (ANOVA) demostró que no existían diferencias significativas entre la media aritmética entre grupos de edades, lo cual, fue corroborado aplicando las correspondientes pruebas de homogeneidad de varianzas.

Considerando que no existen diferencias significativas entre los valores de FEB promedio por edad, se considera que

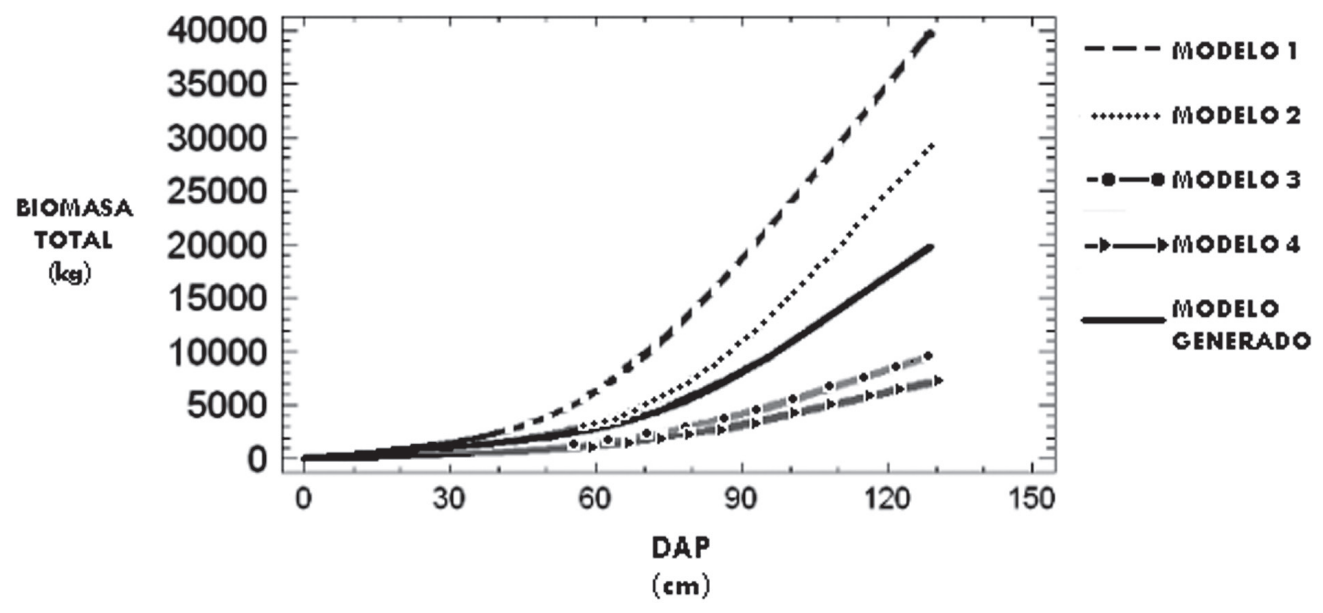

Figura 2. Comportamiento entre las curvas de crecimiento de biomasa para árboles de los modelos reales de campo y del modelo generado por datos simulados.

Cuadro 3. Modelo alométrico genérico para estimar biomasa en los árboles de sombrío.

\begin{tabular}{|l|l|l|l|}
\hline \multicolumn{1}{|c|}{ PARÁMETRO } & \multicolumn{1}{c|}{ MODELO } & $\mathbf{R}_{\text {ajust. }}^{z}$ & \multicolumn{1}{c|}{ RCME } \\
\hline BIOMASA TOTAL & $\ln \left(\mathrm{B}_{\mathrm{t}}\right)=2,44476 * \ln ($ dap $)-1,98675$ & 0,87 & 0,74 \\
\hline Bt = Biomasa total $(\mathrm{kg})$; dap $=$ diámetro a la altura del pecho $(\mathrm{cm})$; ln $=$ logaritmo base e \\
\hline
\end{tabular}


el valor de FEB para los árboles que componen el sombrío de cafeto, corresponde al promedio general de la muestra que, para este caso, fue de 1,93. Este valor coincide con lo expuesto por Andrade E Ibrahim (2003), quienes sostienen que en sistemas agroforestales es posible encontrar valores promedios de FEB entre 1,9 a 2,4, lo que contribuye a sustentar la hipótesis con respecto a que el patrón de producción de biomasa en sistemas arbóreos, que se desarrollan a "cielo abierto", es mayor en comparación con aquellos individuos que crecen bajo condiciones de sombra.

Modelo alométrico genérico para los árboles de sombrío: El análisis de regresión de los datos transformados generó una ecuación (Cuadro 3) que presentó un coeficiente de determinación ajustado, que explica el $87 \%$ de la variabilidad de la biomasa aérea $\left(R^{2}{ }_{\text {ajust. }}=0,87\right)$ y un error estándar de estimación (RCME) de 0,74 , que define la bondad de ajuste del modelo; no obstante, fue necesario definir rangos de certeza, debido a que los datos fueron construidos por simulación y no reflejan necesariamente el comportamiento real de la variable que es analizada. Considerando la cantidad de datos con los que se construyó el modelo, se definieron niveles de pronóstico, de acuerdo a los rangos de diámetro.

Con base en el modelo, se puede establecer que un árbol con DAP promedio de $40 \mathrm{~cm}$ puede presentar una cantidad de biomasa seca, de aproximadamente $1,13 \mathrm{t}$, resultado que guarda semejanza al emplear el mismo diámetro con el segundo modelo propuesto por Segura E Andrade (2008); aunque también hay que advertir que dicho resultado es mucho mayor que el arrojado por los modelos tres y cuatro para este mismo valor diamétrico, lo cual, resulta perfectamente justificable, si se tiene en cuenta que más del $50 \%$ de los árboles de la muestra presentaron valores de biomasa fustal, que superaron el valor de biomasa total pronosticado por dichas ecuaciones, para un diámetro determinado (Figura 2).

Cuantificación de la biomasa total en fincas cafeteras bajo esquemas de certificación: La biomasa aérea total del sistema agroforestal comprende la suma de la biomasa total del cultivo de cafeto y la biomasa de los árboles de sombrío. Debido a la complejidad de los arreglos agroforestales, en términos de especies, estructura y edad, es difícil establecer con certeza la producción potencial de biomasa seca, la que dependerá, básicamente, de la etapa de desarrollo en que se halle cada uno de sus componentes.

Usualmente, en el caso de los árboles maderables, su periodo de vida dentro del sistema agroforestal, se puede extender hasta 20 años antes de ser aprovechados. En el caso del cafeto, se observa que el periodo de vida de un cafetal es de aproximadamente cinco años, antes de que se efectúe la renovación del mismo.
Considerando lo anterior, la biomasa aérea del sistema agroforestal puede fluctuar entre un dato mínimo y uno máximo por hectárea. El valor mínimo contempla que, tanto el cafetal como el sombrío, se siembran al mismo tiempo y, por ende, al cabo del primer año, la cantidad de biomasa aérea será del orden de $2 \mathrm{t} \cdot \mathrm{ha}^{-1}$. De otro lado, el valor máximo se presentará si, tanto el cafetal como el sombrío, coinciden en su edad límite (20 años para los árboles y cinco años para los arbustos de cafeto), lo cual, resultaría en una producción de biomasa de 231t $\cdot$ ha $^{-1}$. Estos valores difieren con los encontrados en otras investigaciones. Suárez et al. (2002) indican que la producción de biomasa en un cafetal joven con sombrío diversificado, menor de cinco metros de altura, puede alcanzar la cifra de $1,1 \mathrm{t} \cdot \mathrm{ha}^{-1}$, mientras que un cafetal productivo con sombrío de más de diez metros, cuantificaría una cantidad de biomasa aérea, del orden de 35,4t·ha-1. En Sumatra, van Noordwijk et al. (2002) reportan existencias promedio de biomasa aérea, del orden de $48 \mathrm{t} \cdot \mathrm{ha}^{-1}$ a $69 \mathrm{t} \cdot \mathrm{ha}$ ${ }^{1}$, para cafetales bajo sombrío, con edades que oscilan entre 2 a 40 años. Concha et al. (2007) señalan que un sistema agroforestal de cacao bajo sombrío, en edades entre cinco a doce años, presentaban existencias de biomasa, que fluctuaban entre 12 a $35,5 \mathrm{t} \cdot \mathrm{ha}^{-1}$, respectivamente.

Es probable que estas diferencias, respecto a los datos presentados en esta investigación, sean el resultado de las variaciones en las prácticas de manejo agronómico, en términos de densidades de siembra, intensidad y composición del sombrío y la edad de los componentes, que conforman el sistema agroforestal.

Se concluye, que la elaboración de modelos alométricos genéricos de biomasa a partir de datos simulados es una alternativa fiable para determinar el crecimiento individual, tanto de árboles que sirven de sombrío como para el cultivo principal (cafeto o cacao), dado que al ponderar los valores de modelos desarrollados con datos obtenidos directamente en campo, se analizan las diferentes tendencias de crecimiento y se adopta un modelo que concilie las características de desarrollo de las especies, como expresión del entorno, en el que se establecen con la probabilidad de generar la cantidad de fitomasa que más se ajuste a la realidad. De otro lado, la construcción de modelos alométricos de biomasa basados en datos simulados, ofrece una alternativa costo-efectiva a los muestreos destructivos cuando esta última no es posible llevarse a cabo, principalmente, en aquellos escenarios en donde el componente arbóreo es conformado por múltiples especies con falencias en información asociada a tasas de producción de biomasa y es imposible desarrollar modelos específicos.

No obstante, el desarrollo de estos modelos está supeditado a la ejecución previa del correspondiente inventario en campo, para determinar datos de volumen individuales. Finalmente, es necesario contar con información de densidad básica 
de las especies involucradas y un factor de expansión de biomasa (FEB) apropiado. En este último caso, se propone emplear la ecuación de FEB desarrollada para el cafeto cuando se trate de este cultivo, en cambio para cacao será necesario desarrollar una ecuación propia. Para el caso de los árboles, se considera apropiado aplicar un FEB fijo de 1,9.

Agradecimientos: El autor agradece la colaboración dispensada por los caficultores del municipio de San Vicente de Chucurí (Santander), quienes hicieron posible que la presente investigación se llevara a cabo. Conflicto de intereses: La presente obra es propiedad intelectual exclusiva de quien funge como autor y quien declara la inexistencia de conflicto de intereses con terceros que pueda poner en riesgo la validez de los resultados presentados. Financiación: Esta investigación fue financiada por COLCIENCIAS y la Fundación Natura.

\section{BIBLIOGRAFÍA}

1. ACERO, L. 1985. Árboles de la zona cafetera colombiana. Ediciones Fondo Cultural Cafetero (Bogotá). 286p.

2. ALBRECHT, A.; KANDJI, S. 2003. Carbon sequestration in tropical agrofrestry systems. Agriculture, ecosystems and environment [en línea]. 99: 15 - 27. Disponible desde internet en: http://faculty.unlv.edu/abellas2/ Restoration_course/Albrecht\%202003\%20C\%20 storage $\% 20$ in $\% 20$ tropical\%20agoforestry $\% 20$ systems\%20Ag\%20Eco\%20Envt.pdf (con acceso el 20/08/2010).

3. ANDRADE, H.; IBRAHIM, M. 2003. ¿Cómo monitorear el secuestro de carbono en los sistemas silvopastoriles? Agroforestería en las Américas (Costa Rica). 10(39,40):109-116.

4. BIRDSEY, R. 1992. Carbon storage and accumulation in United States forest ecosystems. USDA Forest service - Northeastern forest experiment station (USA). 51p.

5. CONCHA, J.; ALEGRE, J.; POCOMUCHA, V. 2007. Determinación de la reservas de carbono en la biomasa aérea de sistemas agroforestales de Theobroma cacao L, en el Departamento de San Martín, Perú. Ecología aplicada (Lima). 6(1-2):75-82.

6. GOLDMAN, C.; SNELL, R.; THOMASON, J.; BROWN D. 1990. Principles of allometry [en línea]. Association for Biology Laboratory Education. Toronto (Canadá). ABLEWEB. 1990. Disponible desde internet en: http://www.ableweb.org/volumes/vol-11/4-goldman. pdf (con acceso el 13/08/2010).
7. INTERGOVERNMENTAL PANEL ON CLIMATE CHANGE (IPCC). 2006. Directrices del IPCC para los inventarios nacional de gases de efecto invernadero [En línea]. IPCC (Geneve). Versión en español. 4(4):93p. Disponible desde internet en: http://www. ipcc-nggip.iges.or.jp/public/2006gl/vol4.html (con acceso el 12/02/2010).

\section{INTERGOVERNMENTAL PANEL ON CLIMATE CHANGE} (IPCC). 2003. Orientación del IPCC sobre las buenas practicas para UTCUTS [En línea]. IPCC (Geneve). Versión en español. 3:325p. Disponible desde internet en: http://www.ipcc-nggip.iges.or.jp/public/gpglulucf/ gpglulucf.html (con acceso el 12/02/2010).

9. OELBERMANN, M.; VORONEY, R.; GORDON, A. 2004. Carbon sequestration in tropical and temperate agroforestry systems: a review with examples from Costa Rica and Southern Canada. Agriculture, Ecosystems and Environment (Amsterdam). 104(3):359-377.

10. ORTIZ, A.; RIASCOS, L.; SOMARRIBA, E. 2008. Almacenamiento y tasas de fijación de biomasa y carbono en sistemas agroforestales de cacao (Theobroma cacao) y laurel (Cordia alliodora). Agroforestería en las Américas (Costa Rica). (46):2629.

11. PEARSON, T.;BROWN, S.;BIRDSEY, R. 2007. Measurement guidelines for the sequestration of forest carbon. USDA Forest service (Newton Square - USA). 42p.

12. PEARSON, T.; WALKER, S.; BROWN, S. 2005. Sourcebook for land use, land - use change and forestry projects. BioCarbon Fund - Winrock International (Washington D.C). 57p.

13. RAINFOREST ALLIANCE. 2009. Norma para agricultura sostenible. Red de agricultura sostenible - Rainforest Alliance (San José, Costa Rica). 42p.

14. ROMINJ, M.; WILDERINK, E. 1987. Fuelwood yield from coffee prunings in the Turrialba valley. En: Somarriba, E. ed. Investigación agroforestal del proyecto UNU/ CATIE 1979 - 1987. Centro Agronómico Tropical de Investigación y Enseñanza (CATIE), (Turrialba, Costa Rica): p.53-71.

15. SEGURA, M.; ANDRADE, H. 2008. ¿Cómo construir modelos alométricos de volumen, biomasa o carbono de especies leñosas perennes? Agroforestería en las Américas (Costa Rica). (46):89-96. 
16. SEGURA, M.; KANNINEN, M.; SUAREZ, D. 2006. Allometric models for estimating aboveground biomass of shade trees and coffee bushes grown together. Agroforestry systems (Netherlands). 68(2):143-150.

17. SUÁREZ, D.; STONIAN, D.; SEGURA, M.; HAGGAR, J.; LOCATELLI, B.; GÓMEZ, M. 2002. Cuantificación y valoración económica del servicio ambiental almacenamiento de carbono en sistemas agroforestales de café en Yassica sur, Nicaragua. Centro Agronómico Tropical de Investigación y Enseñanza (CATIE) (Turrialba, Costa Rica). 8p. Disponible en: http://www.geolatina.net/cuencasnicaragua/sites/ default/files/file/Cap\%204/Cuantificacion\%20y\%20 valoracion\%20C\%20almacenado\%20en\%20SAF.pdf (con acceso el 23/08/2010).
18. VALLEJO, A.; HERNÁNDEZ, P.; De JONG, B.; MARTíNEZ, C.; RODRÍGUEZ, P. 2008. Preguntas frecuentes sobre la estimación de carbono para proyectos forestales MDL. Salinas, Z.; Hernández, P. eds. Guía para el diseño de proyectos MDL forestales y de bioenergía. CATIE - CIFOR (Turrialba, Costa Rica). p.43-66.

19. Van NOORDWIJK, M.; RAHAYU, S.; HAIRIAH, K.; WULAN, Y.; FARIDA, A.; VERBIST, B. 2002. Carbon stock assesment for a forest to coffee conversion landscape in Sumber - Jaya (Lampung, Indonesia): from allometric equations to land change analysis. Science in China (series C) (Beijing, China). 45(s1):75-86.

Recibido: Abril 9 de 2010

Aceptado: Marzo 14 de 2011 